\section{Intersections}

Canadian Journal of Music

Revue canadienne de musique
Intersections CANADIAN JOURAL OF MUSIC
REVUE CANADIENEE DE MUSIQUH

\title{
Littérature et vocalité chez Xenakis ou comment traiter des abîmes
}

\section{Nicolas Darbon}

Volume 34, numéro 1-2, 2014

URI : https://id.erudit.org/iderudit/1030872ar

DOI : https://doi.org/10.7202/1030872ar

Aller au sommaire du numéro

\section{Éditeur(s)}

Canadian University Music Society / Société de musique des universités canadiennes

\section{ISSN}

1911-0146 (imprimé)

1918-512X (numérique)

Découvrir la revue

Citer cet article

Darbon, N. (2014). Littérature et vocalité chez Xenakis ou comment traiter des abîmes. Intersections, 34(1-2), 113-134. https://doi.org/10.7202/1030872ar

\section{Résumé de l'article}

Le mythème du Chaos est étudié à travers les oeuvres vocales de Xenakis. Il est représentatif de l'histoire, des préoccupations et de la sensibilité " contemporaines " (de la fin du $\mathrm{XX}^{\mathrm{e}}$ siècle) : bien que s'inspirant de l'Antiquité grecque, le traitement du texte oscille entre les pôles de l'abstraction pure et de l'expression signifiante. Furieux, tempétueux, le chaos xenakien peut résulter du traumatisme, de la folie, de la guerre, désigner l'état de ce qui n'a pas de forme, la béance, le déluge, la genèse, la perdition, être transition, processus, représenter l'Origine ou la Fin du monde. Ainsi le compositeur a-t-il voulu " traiter des abîmes " par la vocalité soliste ou chorale, remonter à « toute la richesse cosmique, mais chaotiquement ». Cet article ouvre un diptyque dont le second volet s'intitule " La Grande Mère néolithique dans Serment-Orkos » (publié par le Centre Iannis Xenakis, Université de Rouen). Le postulat est le suivant : les sciences dites du " chaos » et de la " complexité » relèvent elles-mêmes d'une anthropologie de l'imaginaire. Par ailleurs, il s'inscrit dans un champ de recherche sur la transdiction (notion explicitée sur le site du CEREdI, Université de Rouen), c'est-à-dire sur le son et la corporéité des transferts musico-littéraires.
Copyright ( C Canadian University Music Society / Société de musique des universités canadiennes, 2015
Ce document est protégé par la loi sur le droit d'auteur. L’utilisation des services d'Érudit (y compris la reproduction) est assujettie à sa politique d'utilisation que vous pouvez consulter en ligne.

https://apropos.erudit.org/fr/usagers/politique-dutilisation/ 


\title{
LITTÉRATURE ET VOCALITÉ CHEZ XENAKIS OU COMMENT TRAITER DES ABÎMES
}

\author{
Nicolas Darbon
}

Chez Xenakis, le poème à la source de l'inspiration, sa musicalité interne et sa prosodie, peuvent disparaitre ou n'être que trace. À tel point que l'utopie d'une source originelle l'emporte sur les possibles méandres du texte et de la langue. D’autant que la musique xenakienne s'origine dans le puissant fond littéraire de l'Antiquité grecque. La présente étude portera sur la notion de traduction musicale et se limitera aux œuvres vocales de Xenakis. Toutefois, il semble difficile d'imaginer traduire quoi que ce soit en musique lorsque le compositeur, qui est aussi architecte, se déclare «abstrait» (1994, p. 158-164), prônant ce qu'on pourrait appeler une forme de "musique pure». Xenakis oscille entre le pôle de l'abstraction et celui de la signification.

Les archétypes du chaos me paraissent déterminants dans cette problématique. Déjà, sur l'île grecque de Spetses, jeune homme farouche et solitaire, Xenakis communiait avec les forces primordiales ${ }^{1}$. Plus tard, le Polytope de Mycènes (1978), qui est une sorte de "son et lumière", lâche deux cents chèvres portant des cloches dans une vallée appelée Chaos. Furieux, tempétueux, le chaos peut résulter du traumatisme, de la folie, désigner l'état de ce qui n'a pas de forme, être transition, processus, représenter l'origine ou la fin du monde. Le compositeur qui, résumant sa démarche, déclare qu'il a "voulu traiter des abîmes» (1978/2006, p. 353) n'est pas sans connaître les théories du chaos, mais à la science pure il adjoint de profondes motivations imaginaires. C'est pourquoi, sans trop de contrainte, je me suis demandé ce que Xenakis avait proposé. La société telle qu'elle s'exprime, ce que Maffesoli appelle la forme contemporaine, comporte un versant antiorphique (Darbon, 2007b). «Au travers des rêves collectifs, des mythes et des archétypes, c'est toute la préhistoire de l'humanité qui continue de s'exprimer.» (Maffesoli, 1996/2005, p. 135) Il s'agit donc d'aller à la découverte de ces résurgences/émergences de pulsions archaïques telles qu'elles sonnent chez Xenakis.

Cette étude développera donc deux axes méthodologiques : la transdiction ${ }^{2}$, à savoir le transfert de la littérature (l'empreinte sonore originelle) vers la musique; et l'archéo/sociomusicologie, dans l'esprit du «formisme» de Michel Maffesoli (1985).

1 «J'aimais infiniment la nature. J'allais en bicyclette à Marathon. À l'endroit supposé de la bataille, il y avait un tumulus avec un bas-relief d'Aristoclès, et là je restais longtemps à m'imprégner des bruits de la nature, des cigales, de la mer.» (1980/2006, p. 17).

2 Pour une définition, consulter mon article : "Qu'est-ce que la transdiction?», accessible en ligne : http://ceredi.labos.univ-rouen.fr/public/?qu-est-ce-que-la-transdiction.html. 


\section{LA FURIA OURANIENNE}

L'invasion de la péninsule par les Grecs, peuple indo-européen, a apporté aux civilisations égéennes en place des figures masculines, comme celle d'Ouranos ou de Zeus. La manifestation musicale du chaos ouranien passe par les schèmes dynamiques et violents des éléments naturels, et de l'homme en lutte contre les éléments. Ce corps à corps avec la matière-monde qui gronde et soulève les émotions les plus fortes, Xenakis l'a recherché lorsqu'il s'immergeait dans la nature. Peut-être néglige-t-on que le Grec est une créature de la roche et de l'océan, de la fournaise et du bleu marin, créature de la nature plutôt que citadine, lui, le citoyen exilé en terre parisienne, prisonnier de la carrière qu'il s'impose, celle d'architecte et de compositeur d'avant-garde. À partir de 1951, peu de temps après avoir pris souche à Paris, il décide de passer chaque été quatre semaines en Corse avec sa femme et sa fille. Ils campent face à la mer, dans des conditions spartiates, seuls, dans une petite tente, perdus, loin des vaches, des chèvres, des humains. Sa fille, Mâkhi raconte ainsi qu'"il attendait les orages d'été et lorsque la foudre et les grondements de tonnerre arrivaient juste au-dessus de nos têtes, il se mettait à courir en haut de la montagne pour se retrouver au cœur de l'orage.» (Mâkhi Xenakis, 2011, n.n.) La phrase qui termine ce témoignage est stupéfiante, elle montre un aspect fantastique du personnage : «Son visage alors se détendait et il semblait profondément heureux...». Aimanté par le tonnerre et l'ouragan, Xenakis accède à l'apaisement et au bonheur ! À propos de Terretektorh, Xenakis demande que l'auditeur soit "perché sur le sommet d'une montagne au milieu d'une tempête l'envahissant de partout» ou "sur un esquif frêle en pleine mer démontée ", ou encore "dans un univers pointilliste d'étincelles sonores, se mouvant en nuages compacts ou isolés.» (disque Erato STU70529).

\section{LE DÉLUGE PRIMORDIAL}

Au commencement, le chaos signifie le déluge, la noyade, l'engloutissement, l'inondation, la destruction-purification, le génocide-renaissance. L'un des plus vieux textes est sumérien; il date du XVIII siècle AC: la Genèse d'Eridu relate un évènement historique, la formation de la Mer Noire à travers un gigantesque raz de marée. D’où la figure récurrente de l'abîme et du chaos - version déluge - dans les récits mythologiques. Xenakis défie cet élément. "Ce qu'il aimait le plus » poursuit Mâkhi «était d'attendre une tempête afin de pouvoir partir en kayak l'affronter.» Xenakis n'est pas un contemplatif timoré; il ne reste pas sur la montagne pour laisser passer au-dessus de lui les éclairs; il s'engouffre dans la mer déchaînée! «Ma mère bien sûr avait très peur, mais ni ses pleurs ni ses menaces ne le faisait changer d'avis.» Il gardait une confiance inébranlable en ses forces et sa supériorité, au point d'emmener sa fille dans l'embarcation, qui, par fierté et par bravade, le suivait. «Et nous nous retrouvions au milieux des vagues en furies à ramer comme des forcenés au milieu de l'écume pour éviter d'être retournés par une vague exceptionnelle... et écrasés sur les rochers...» Lui qui a été de nombreuses fois emprisonné pendant la guerre et a côtoyé la mort de près, continuait de la tutoyer en allant en kayak 
"d'une île à l'autre dans l'Egée», se souvient François-Bernard Mâche, «un défi hérö̈que, et l'expérience d'un tumulte sonore dont il a livré dans plusieurs partitions une poétique transmutation.» (Mâche, 2012, cf. aussi Françoise Xenakis, 1979).

\section{L'INFORMité, LE PASSAGE, LA MÉTAMORPHOSE}

Eboulis, tas, amas, poudroiement, fouillis. Le propre du chaos est d'être informe. Sens dérivé : inanimé; la vie suppose l'organisation; le chaos c'est la mort; non pas le néant, mais ce qui n'est pas encore, ou ce qui n'est plus ; l'inerte, le pulvérisé. Ovide dans ses Métamorphoses [I, vers 6-7] donne au chaos cette signification de masse grossière, inorganisée. L'informité musicale réside par exemple dans l'indétermination et l'imprécision volontaire de l'écriture. «La musique n'est pas une langue» (1978/2006c, p. 353), elle n'est pas pour Xenakis une structure de significations, au contraire. L'informe vocal se présente par exemple au début d'Orestïa [L'Orestie] (1965-66), pour chœur d'enfants, chœur mixte et ensemble, par une notation dont la portée est réduite à deux barres horizontales imprécises sur lesquelles des vagues suggèrent des hurlements, un graphisme pointilliste propose un nuage de sons. Les deux mezzo-sopranos de N'Schima (1975, p. 7) doivent choisir leurs hauteurs dans une zone large et indécise. Cendrées (1976, p. 7), pour chœur mixte et orchestre, torsade un nuage de sons-phonèmes (Ex. 1).

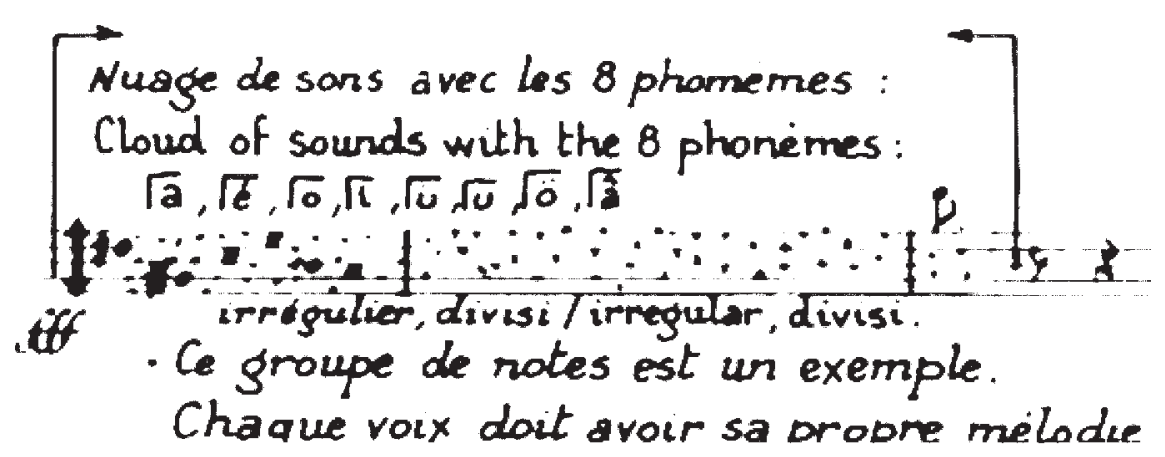

Ex. 1. Cendrées (1973), pour chœur mixte et orchestre, extrait p. 7, sopranos en tutti.

Un procédé présent dans Serment-Orkos (1981) pour chœur mixte a cappella est très ancien et bien connu, c'est le glissando des hauteurs (qui est en réalité dépendant du temps : Barthel-Calvet, 2006)3. Le glissando vocalique participe du même esprit; voyez dans la même œuvre (p. 1) le passage d'une voyelle à une autre, $d u$ « $a » a u$ «e», ou encore $d u$ « $u$ » $a u$ « $\mathrm{o} »$ en passant par le «a» (Ex. 2).

3 «Dès 1956, Xenakis associe la "notion mathématique de vitesse» au déplacement continu du son dans l'espace hauteurs-temps, c'est-à-dire au glissando. (...) L'assimilation géométrique du glissando à une ligne va permettre un transfert poiétique de structures graphiques dans l'univers sonore qui va faire émerger des configurations proprement inouïes.» (Barthel-Calvet, 2005). 


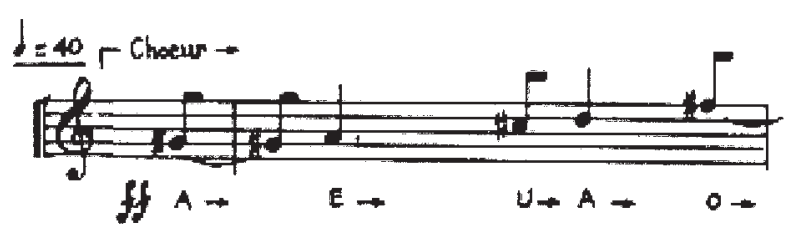

Ex. 2. Serment-Orkos, 1981, p. 1.

Cependant à l'audition, le résultat est peu perceptible, en raison du tempo assez rapide.

Les deux types de glissandos, sonique et vocalique, peuvent se combiner (Ex. 4). L'utilisation et même l'invention de phonèmes sont des classiques de Xenakis. Partant du principe que pour « rendre musical un texte (...) on peut inventer ses propres phonèmes", Nuits (1967-68) pour chœur mixte convoque des sons trouvés chez les sumériens, chez les persans, les japonais. Le sens du texte est brouillé, d'autant que l'harmonie est glissante et micro-intervallique. Christine Prost (1981, p. 280-281) explique bien l'efficacité de procédés «informels » dans l'Orestie (1965-66), pour chœur d'enfants, chœur mixte et ensemble. L'organisation métrique du discours n'est pas exacte, mis à part la référence à une certaine durée, ce qui lui confère «une souplesse et une dynamique extrême». D'ailleurs, «l'absence de direction du chef (...) investit les interprètes d'une responsabilité collective, stimulante, voire exaltante». Les quarts et tiers de ton s'opèrent par approximation et ajustement collectif, car l'effort de justesse parviendrait à l'effet inverse, la crispation. "Cette imperfection n'occulte pas le rayonnement d'une musique que l'on ressent comme l'expression de forces primordiales, une musique puissante, qui met en jeu l'être total de celui de l'interprète.»

De manière générale, l'informité trouve un terrain favorable dans les masses sonores, quand la globalité l'emporte. Pièce de jeunesse, le Sacrifice (1953), pour orchestre, est un extrait des Anastesaria. Elle illustre cette tendance à la globalité sonique à tous les niveaux :

a. Globalité sonique au niveau micro. Par exemple, les glissandos et micro-intervalles sont signalés grâce à un signe de la notation byzantine, afin de "produire des interférences». D’où tout un jeu sur les battements, proches des turbulences en théorie du chaos.

b. Globalité sonique au niveau macro, celui de l'œuvre.

Précisons aussi l'importance du hasard, de l'aléatoire, qui brouille les pistes, tels les mouvements browniens ${ }^{4}$ dans les deux pièces vocales déjà citées, Cendrées et N'Schima, conçues à l'aide d'un ordinateur. Ainsi les glissandos sont

4 Pour Xenakis, le mouvement brownien est «l'image, projetée sur diverses dimensions, d'une particule, d'un point abstrait, théorique et mathématique, qui se soumet aux lois probabilistiques ». Parmi les œuvres instrumentales utilisant le modèle brownien lié aux glissandos : Mikka (1972), Mikka S (1976), Theraps (1976). (Restagno, 1988, p. 50). 
l'empreinte des particules en plein mouvement brownien. Il existe donc un type de glissando non linéaire.

Peu de fixité : ainsi la métaforme (forme glissante) côtoie la forme. Janus, le dieu aux deux visages, gardien des passages et des croisements, divinité du changement : son premier nom était Chaos 5 . Le chaos est donc ambiguïté, passage, processus et métissage, mais aussi confusion, perfidie, mensonge : comme Bel ou Baal le confondeur, Koush - dont la racine signifie aussi bien le chaos que le caché - est le seigneur des dissimulations et de la confusion. Les procédés précités (glissandos, etc.) sont autant de passages. L'écriture par processus de transformation est très visible dans Serment-Orkos (p. 25). Les effets de masse ont besoin d'un orchestre et de la division extrême des parties, davantage que d'un ensemble vocal réduit. Parmi les pièces orchestrales, Kyania (1990) réutilise des matériaux de Horos (1986/89), laquelle développe la théorie des Automates Cellulaires ${ }^{6}$, comme le fait un autre pionnier en la matière, György Ligeti ${ }^{7}$, principe de propagation végétale, des lichens par exemple (titre d'une pièce de 1984). «En suivant des règles très simples, il est possible de donner vie à un parcours qui se développe progressivement» (Restagno, 1988, p. 61), explique Xenakis. Ce sont donc des règles de propagation : à partir d'une cellule originelle, une texture se développe de proche en proche. Xenakis indique quatre modalités de propagation : 1) dans la simple ligne mélodique; 2) dans la masse orchestrale; 3) d'un accord vers l'autre ; 4) d'une couleur vers l'autre. Autre théorie, celle des probabilités. Elle implique un grand nombre d'événements désordonnés et imprévisibles, formant des galaxies acoustiques, transposition de l'architecture à la musique de lignes droites ou courbes, à l'archet ou en pizzicato (Pithoprakta, 1956, pour orchestre à cordes). Les notes sont assimilées à des molécules obéissant aux lois de la mécanique des fluides, comme un gaz sous pression contenu dans un lieu clos. La vitesse des glissandos est assimilée à celles des molécules de gaz (1963, p. 30). En somme, la métaforme est présente de la stochastique aux modèles des sciences de la nature, comme ceux de la théorie des catastrophes.

L'expérience des processus chaotiques est relatée dans un écrit célèbre de Xenakis, faisant un lien entre phénomènes naturels, politiques et musicaux (1962/1994, p. 58). Il décrit une foule politisée; un mot d'ordre lancé en tête se propage jusqu'à la queue, à l'instar d'une onde de transition. Dans ce fleuve humain, se produit alors un choc entre flux régulé (les pas et les chants) et turbulence (lorsqu'une perturbation policière survient par exemple) :

Le rythme parfait du dernier mot d'ordre se rompt en un amas énorme de cris chaotiques qui, lui aussi, se propage à la queue. (...) Les lois statistiques de ces événements vidés de leur contenu politique ou moral sont celles des cigales ou de la pluie. Ce sont des lois de passage de l'ordre

5 «Me Chaos antiqui, man, res sum prisca, vocabant.» (Ovide, rééd. 1990, p.19).

6 Autre pièce instrumentale citée par Xenakis utilisant les Automates Cellulaires : Ata (1987). Sur les Automates Cellulaires dans la musique de Xenakis. (Hoffmann, 2006, p. 145-52 ; 1994, p. 145152 ; 2002, p. 121-131. Xenakis/Restagno, 1988, p. 61. Varga, 1996, p. 182-184, 199-200. Gibson, 2003, p. 166-168. Harley, 2004, p. 176-178. Solomos, 2006).

7 Dans Melodien (1971) pour petit orchestre par exemple (Darbon 2006, p. 76-79). 
parfait au désordre total, d'une manière continue et explosive. Ce sont des lois stochastiques. Ici nous touchons du doigt un des grands problèmes qui ont hanté l'intelligence de l'Antiquité; la transformation continue ou discontinue.

\section{LE FRACAS, LE TRAUMA, LA DÉMENCE, LA PERDition}

Cette foule humaine explosée sous le fracas des balles, Xenakis l'a vécue pendant la guerre, c'était une manifestation antinazie à Athènes. Si cet événement, «hautement puissant et beau dans sa férocité» (ibid.), le marque, c'est aussi pour sa valeur "esthétique». La clameur se désagrège en un «chaos énorme » quand se mêlent les sifflements de balles, le vacarme des mitrailleuses, qui font songer aux phénomènes naturels comme "les lames qui se brisent contre les falaises, les déferlements des vagues qui se brisent sur les galets.» Comme le dit sa fille : "mon père reviendra souvent sur les mouvements de masse des manifestants, les coups de canons, de feux, les cris des blessés et la nuit où sous les projecteurs et les feux, ces moments devenaient des spectacles uniques....»Plus loin, sa fille Makhi explique comment son père a été défiguré :

En 1944 alors que les Anglais commencent à attaquer un immeuble et que mon père, en tant que chef de résistance, vient de mettre les habitants au sous-sol il reçoit avec ses camarades, des coups de mortier, une jeune femme à côté de lui est atteinte par un obus, des traces de cervelles recouvrent les murs de la pièce, mon père juste à côté est touché au visage très violemment son œil gauche arraché. (...) Sa compagne, Makhi viendra à ses côtés, plus tard quand les Anglais lui enlèveront l'éclat d'obus de son visage, elle ira le perdre dans la montagne...

Il faudrait séparer quatre catégories chaotiques coagulées dans ce mythème :

- le fracas, la tellurie, la sauvagerie, les dieux Pan, Dionysos

- le traumatisme de l'histoire individuelle et collective

- la folie, l'homo demens, l'hubris

- la perdition, la divagation.

La guerre les réunit toutes.

La perdition se retrouve dans des applications des théories du «chaos», du hasard, comme le mouvement brownien, l'entropie, dans de multiples œuvres de Xenakis; il s'agit de se plonger aussi dans un état plus ou moins «intemporel», un lieu comme disait Ovide sans orientation, où l'on va, divague et chute dans tous les sens.

La littérature concentrationnaire ne parvient pas à décrire l'abominable, elle le fait souvent par élision; le chaos «s'écrit classique». Pourtant, chez Xenakis, le cri, la sauvagerie, les gémissements s'entendent. La musique vocale rejoint souvent le bruit vocal : utilisation de nasales, de gutturales, etc., car pour Xenakis les notions de Beau et de Laid n'ont pas de sens. Sauvagerie qui renvoie au 
fracas de l'origine, et non au sentiment, même si Michel Philippot parle de romantisme (Philippot, 1985, p. 1131-33). ${ }^{8}$

La conception assez classique de Serment-Orkos d'après le sage Hippocrate contient aussi - surprise pour un tel texte - un grand cri central (mes. 31-33.) Ecoutez aussi les «cris vocaux» de Nuits, dédiée aux prisonniers politiques; ou les partitions décrivant les massacres d'Agamemnon, Egisthe, Clytemnestre; écoutez encore les «désastres instrumentaux», telles que Phlegra (1975) pour ensemble, du nom du champ de bataille entre les Titans et les dieux de l'Olympe; les hurlements sans phonèmes de l'Orestie (Ex. 3) et ceux mélangés qui se mêlent aux paroles :

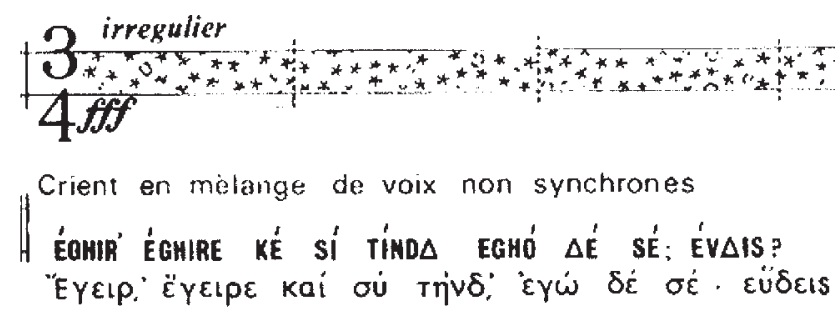

Ex. 3. Cris mélangés : l'Orestie, début, 1981.

\section{L'ORIGINE, LA VÉRITÉ PREMIÈRE}

«L'astrophysicien ne crée pas les galaxies qu'il sonde alors que le musicien est capable de produire les siennes dans son acte créateur.» (1980/2006, p. 23)

Avant la guerre, avant même la construction du monde, avant la «vie», c'est l'explosion, à peine une genèse, une naissance, certains parlent de primitivisme. Contre les sophistications, ce qu'il appelle la «scolastique» de l'immédiat après-guerre, Xenakis est à la recherche de l'origine.

Dans ses carnets de 1951 (lignes 2 à 12), Xenakis écrit ceci :

Voilà la tâche que je dois accomplir cette année. Aller chercher la composition (mélodie, harmonie, rythme, synthèse) en sa demeure secrète et enfouie au plus profond de l'art primitif. Le contraire de la diversité et de la complexité moderne ! L'origine de la musique c'est ça qu'il faut remettre en place.

Si Xenakis oppose chaos et complexité, c'est justement parce que pour lui, avec la diversité, la complexité représente le sérialisme (intellectualisme gratuit), le néoclassicisme, voire les musiques de «variétés» (la diversité superficielle). La vérité originelle de l'Antiquité grecque est dans ce chaos. Dans le domaine

8 La sentimentalité a été réprimée dans les styles d'après-guerre qui prétendaient au modernisme, ce qui explique l'exécration des musiques jazz, amplifiées, néoclassiques, postmodernes ; paradoxalement, on a pu parler de néoclassicisme pour le Xenakis de l'Orestie. 
instrumental, les références à l'Antiquité chez Xenakis (Mâche, 2000, p. 302321) font apparaître des forces contradictoires :

- Les forces inconscientes des pré-olympiens, les Géants, les Titans9.

- Les forces fondatrices de la civilisation ${ }^{10}$.

- Les forces de la mort et de la fortune ${ }^{11}$.

L'œuvre vocale se concentre sur le dernier point. On peut éventuellement ranger dans le deuxième point la Théogonie d'Hésiode qui décrit les fondements de la mythologie grecque. Xenakis tentera, dans l'Orestie, de garder la langue de l'Antiquité qu'il juge «extraordinaire (...) tant du point de vue de la phonétique que de celui des images et de l'invention" (1956/1994, p. 51). Ce qui l'attire chez Eschyle, c'est qu'il incarne un théatre archaïque bien plus «moderne» que les autres» (ibid., p. 52). Xenakis le moderne (postmoderne dirions-nous aujourd'hui) déclare ainsi chercher la reconstitution et la réinvention. "La tragédie antique était pour moi quelque chose de courant» : c'est ce monde qu'il fréquente depuis sa jeunesse. Il cherche par ailleurs le théâtre originel, qu'il place au-dessus de l'opéra, qui n'est qu'un «amalgame», dont les livrets sont secondaires et est un «spectacle factice». Le théâtre antique d'Eschyle, c'est la vérité et la pureté (ibid., p. 53). Ce qu'il appelle le «théâtre total» (poésie, action danse, musique; gestique sobre; symbolisme). L'Orestie utilise l'intonation du grec moderne, mais après Hiketides [Les Suppliantes] (1964) pour chœur de femmes, à partir de A Colone (1977) pour chœur, Xenakis ira plus loin en remontant jusqu’à la phonétique de l’antiquité.

\begin{tabular}{|c|c|c|c|}
\hline \multicolumn{2}{|c|}{ De 1949 à 1977} & \multicolumn{2}{|c|}{ De 1977 à 1994} \\
\hline Guvre vocale & Source littéraire & Fuvre vocale & Source littéraire \\
\hline $\begin{array}{l}\text { I Air populaire } \\
\text { pno-1949 }\end{array}$ & $\begin{array}{l}\text { Chanson populaire } \\
\text { grecque }\end{array}$ & $\begin{array}{l}\text { I A Hélène } \\
\text { Ch de f ou d'h, } \\
10 \text { '-1977 }\end{array}$ & Euripide (Hélène) \\
\hline $\begin{array}{l}\text { I Allegro molto } \\
\text { Pno-1949 }\end{array}$ & $\begin{array}{l}\text { Chanson populaire } \\
\text { grecque }\end{array}$ & $\begin{array}{l}\text { I Akanthos } \\
\text { Sop, octuor, 11'-1977 }\end{array}$ & \\
\hline $\begin{array}{l}\text { I Mélodie } \\
\text { Pno-1950 }\end{array}$ & Poème grec & $\begin{array}{l}\text { I A Colone } \\
\text { Ch de f ou d'h (20), ens, } \\
14^{\prime}-1977\end{array}$ & Euripide (Hélène) \\
\hline $\begin{array}{l}\text { I Six chansons } \\
\text { (« Cela sent le musc », } \\
\text { etc.) }\end{array}$ & Poème grec & $\begin{array}{l}\text { I Anémoessa } \\
\text { Ch mixte ( } 42 \text { ou } 84) \text {, } \\
\text { orch, } 15^{\prime}-1979\end{array}$ & \\
\hline $\begin{array}{l}\text { I Tripli zyia, } \\
\text { voix, fl, vc - } 1951\end{array}$ & Poème grec & $\begin{array}{l}\text { I Aïs } \\
\text { Baryton amplifié, percu, } \\
\text { orch.-1980 }\end{array}$ & $\begin{array}{l}\text { Homère (l'Illiade, } \\
\text { l'Odyssée) } \\
\text { Sapho }\end{array}$ \\
\hline $\begin{array}{l}\text { I Zyia } \\
\text { sop, ch. d'h, fl, pn, } \\
\text { 10'-1952 }\end{array}$ & Poème grec & $\begin{array}{l}\text { I Serment-Orkos } \\
\text { Ch mixte (32), 7'-1981 }\end{array}$ & Hippocrate \\
\hline
\end{tabular}

9 Phlega (1975) pour ensemble : les Géants; Kottos (1977) pour violoncelle : les Titans.

10 Atreus dans l'Orestie: Mycènes; Keqrops, (1986) pour piano et orchestre : Athènes.

11 Evryali (1973) pour piano : la Gorgone ; Kassandra (1987) pour baryton et percussion : la Prophétesse. 


\begin{tabular}{|c|c|c|c|}
\hline \multicolumn{2}{|c|}{ De 1949 à 1977 continué } & \multicolumn{2}{|c|}{ De 1977 à 1994 continué } \\
\hline $\begin{array}{l}\text { I Trois poèmes } \\
\text { Vx récitante, pn - } 1952\end{array}$ & $\begin{array}{l}\text { Villon (Aies pitié de } \\
\text { moy) ; Mayakowsky } \\
\text { (Ce soir je donne mon } \\
\text { concert d'adieu) ; Ritsos } \\
\text { (Symphonie printanière) }\end{array}$ & $\begin{array}{l}\text { I Nekuia } \\
\text { Ch mixte (54 ou } 80) \text {, } \\
\text { orch, } 26 \text { '-1981 } \\
\text { I Pour la Paix } \\
4 \text { versions : } \\
\text { - ch mixte,-4 } \\
\text { récitants, ch mixte, } \\
\text { bande,-id, mais c } \\
\text { préenregistré,--bande } \\
\text { seule, contenant le tout. } \\
10^{\prime} / 27 \text { ' }\end{array}$ & $\begin{array}{l}\text { Jean-Paul Richter } \\
\text { (Siebenkäs) } \\
\text { Françoise Xenakis } \\
\text { (Ecoute, Les Morts } \\
\text { pleureront) }\end{array}$ \\
\hline $\begin{array}{l}\text { I La Colombe de la } \\
\text { paix, Alto, ch mixte à } 4 \\
\text { vx-953 }\end{array}$ & Theodosis Pieridis & & \\
\hline $\begin{array}{l}\text { I Anastenaria-1953 } \\
\text { a) Procession vers les } \\
\text { eaux claire }\end{array}$ & $\begin{array}{l}\text { (Culte Thrace de } \\
\text { Dionysos) }\end{array}$ & $\begin{array}{l}\text { I Pour Maurice } \\
\text { Caryton, pn, 4'-1982 }\end{array}$ & \\
\hline $\begin{array}{l}\text { ch mixte (30), ch d'h } \\
\text { (15), orch, 8' } \\
\text { b) Le Sacrifice, orch }\end{array}$ & & $\begin{array}{l}\text { I Chant des soleils } \\
\text { Ch d'enfants, ch mixte, } \\
\text { ens de cuivres, percu, } 8 \\
\text { '-1983 }\end{array}$ & Peletier du Mans (XVIe) \\
\hline $\begin{array}{l}\text { I Stamatis Katotakis } \\
\text { Voix, ch d'h à } 3 \text { vx, } \\
\text { inédit, } 1953\end{array}$ & (Chanson de table) & $\begin{array}{l}\text { I Idmen A } \\
\text { Ch mixte (32), } 4 \text { percu, } \\
14 \text { '-1985 }\end{array}$ & $\begin{array}{l}\text { Hésiode (Théogonie) et } \\
\text { Phonèmes }\end{array}$ \\
\hline $\begin{array}{l}\text { I Polla ta dhina } \\
\text { ch d'enfants (20), orch., } \\
\text { 6' - } 1962\end{array}$ & Sophocle (Antigone) & $\begin{array}{l}\text { I Idmen B } \\
\text { Ch éventuel, } 6 \text { percu, } \\
\text { 14'-1985 }\end{array}$ & $\begin{array}{l}\text { Hésiode (Théogonie) et } \\
\text { Phonèmes }\end{array}$ \\
\hline $\begin{array}{l}\text { I Oresteïa [L'Orestie] } \\
\text { Ch d'enfants jouant } \\
\text { d'accessoires musicaux, } \\
\text { ch mixte (36), ens, } \\
46 \text { '-1966 }\end{array}$ & Eschyle & $\begin{array}{l}\text { I Kassandra } \\
\text { Cycle : Oresteïa, pre- } \\
\text { mière partie Baryton } \\
\text { jouant sur un psal- } \\
\text { térion, cordes, percu, } \\
\text { 11'-1987 }\end{array}$ & Eschyle (Orestie) \\
\hline $\begin{array}{l}\text { I Medea } \\
\text { Senecae [Médée] } \\
\text { ch d'h, cl, fg, tb, vlc, } \\
\text { percu, } 25,-1967\end{array}$ & Sénèque & $\begin{array}{l}\text { I Knephas } \\
\text { Ch mixte (32), 10' } \\
-1990\end{array}$ & \\
\hline $\begin{array}{l}\text { I Hiketides [Les } \\
\text { Supplliantes] } \\
\text { ch de f,-1964 } \\
\text { I Nuits } \\
\text { ch mixte (12), 12'-1968 }\end{array}$ & Eschyle & $\begin{array}{l}\text { I La déesse Athéna } \\
\text { Cycle : Oresteïa, } \\
\text { dernière partie (Les } \\
\text { Euménides) } \\
\text { Baryton, ens, 9'-1992 }\end{array}$ & $\begin{array}{l}\text { Eschyle (Orestie) } \\
\text { Eschyle (Orestie ; les } \\
\text { Euménides) }\end{array}$ \\
\hline $\begin{array}{l}\text { I Cendrées } \\
\text { ch mixte ( } 36 \text { ou } 72) \text {, } \\
\text { orch., } 25^{\prime}-1973\end{array}$ & (Phonèmes) & $\begin{array}{l}\text { I Pu wijnuej we fyp } \\
\text { Ch d'enfants (21), } \\
\text { 10'-1992 }\end{array}$ & Arthur Rimbaud \\
\hline \multirow[t]{2}{*}{$\begin{array}{l}\text { I N'Shima } \\
2 \text { mezzo, } 2 \text { cors, } 2 \text { tb, } \\
\text { vlc, 17'-1975 }\end{array}$} & $\begin{array}{l}\text { (Phonèmes et mots } \\
\text { hébreux) }\end{array}$ & $\begin{array}{l}\text { I Les Bacchantes } \\
\text { Baryton, ch de f (16), } \\
\text { ens, 60'-1993 }\end{array}$ & $\begin{array}{l}\text { Euripide (Les } \\
\text { Bacchantes) }\end{array}$ \\
\hline & & $\begin{array}{l}\text { I Sea Nymphs } \\
\text { Ch mixte (24), 8'-1994 }\end{array}$ & $\begin{array}{l}\text { Shakespeare (La tem- } \\
\text { pête), phonèmes }\end{array}$ \\
\hline
\end{tabular}

Il existe un deuxième ensemble de sources, assez composite, de pays et d'époques variés - du mathématicien Peletier du Mans (1517-82) à la femme du compositeur, l'écrivaine Françoise Xenakis (1930-) - , constitué de textes 
populaires, puis de poètes (Richter, Villon, Mayakowsky, Ritsos, Rimbaud), et plus tard de romanciers ou dramaturges comme Shakespeare.

La traduction musicale procède par :

- courtes phrases (Nekuïa, 1981, pour chœur et ensemble),

- vastes passages textuels (l'Orestie, 1966, pour chœur, avec ensemble),

- mélange de phonèmes et de bribes de texte (N’Shima, 1975, pour 2 mezzo-sopranos et ensemble; Idmen A et B, 1985, pour chœur et ensemble),

- ou rien du tout, que des phonèmes (Nuits, 1968, pour chœur a cappella; Cendrées, 1973, pour chœur et ensemble).

J'appelle mosaïque ${ }^{12}$ l'utilisation variée, synthétique de textures et de techniques vocales et chorales; je schématiserais ainsi l'évolution du traitement vocal chez Xenakis, partant d'un paradigme ordonné pour y revenir, mais de façon synthétique :

\begin{tabular}{lll}
\hline Ordre & Chansons populaires & 1949 \\
(prosodie / mélodie) & Anastenaria & 1958 \\
& Polla ta dhina & 1962 \\
& Hiketides & 1964 \\
& Oresteia & 1966 \\
Chaos & Medea Senecae [Médée] & 1968 \\
(bruits / phonèmes) & Nuits & 1968 \\
& Cendrées & 1973 \\
& N'Shima & 1975 \\
Ordre / Chaos & Nekuia & 1981 \\
(prosodie / mosaïque) & Idmen & 1985 \\
& Serment-Orkos & 1981 \\
& Sea Nymphs & 1994 \\
\hline
\end{tabular}

Serment-Orkos utilise des extraits courts du serment et des phonèmes. Ainsi est-ce moins le texte que son Idée-source qui est mise en musique; pour Xenakis, il s'agit de partir du matériau sonore pour aboutir à une globalité. L'affleurement des bribes de ce texte apporte l'aura de cette matière que le compositeur ne veut pas dissoudre. Par affleurement, il faut comprendre un surgissement du sens momentané. Par conséquent, tout un monde en-deçà constitue l'essentiel de cette musique "originelle». Pour Serment-Orkos, il faut s'imaginer la surprise et la perplexité du public au moment de la création. Il s'agissait d'auditeurs venus à un congrès cardio-vasculaire où se mêlaient les lauréats du doctorat de médecine ! Face à eux, une version «sauvage» pour le moins inattendue du serment d'Hippocrate...

Pour donner un effet de parole lointaine, pour colorer du mythe des origines, les ficelles sont parfois banales, comme la scansion du texte. Par exemple, Xenakis utilise dans sa Procession vers les eaux claires une énonciation syllabique à partir d'une vieille mélodie simplifiée - un chant akritique de l'époque bysantine, relatif à la capture d’Amorion par les Arabes en 838 l'Épire ${ }^{13}$.

12 Terme utilisé à propos de Sea Nymphs par Harley, op. cit., p. 237.

13 «Le chant du chœur mixte est basé sur une mélodie akritique [moyenâgeuse] Le château d'Oria d'origine cappadocienne. Mélodiquement, il s'amplifie d'abord puis se simplifie pour aboutir à la page 27 à des permutations de 4 sons, transposées en quartes ou tierces avec entrées successives 


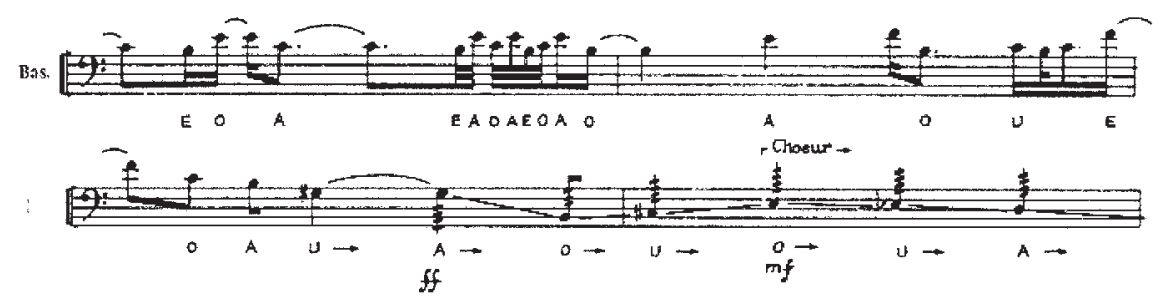

Ex. 4. Serment-Orkos, 1981, mes. 17-22, basse.

Au-delà de la prosodie, Xenakis garde de ses premières compositions d'allure populaires grecques, y compris dans ses partitions «abstraites» ou dans Ser-

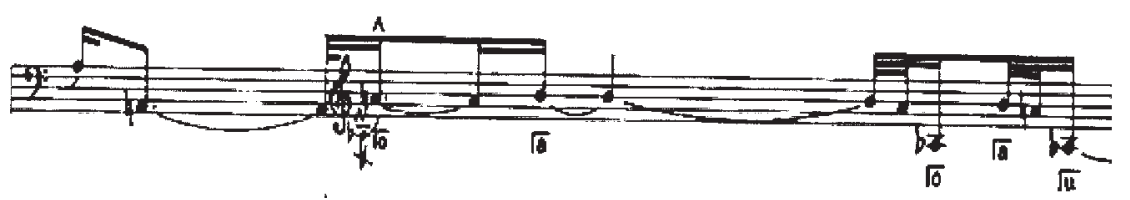

Ex. 5. Pour Maurice (1982), « temps » 73-76, baryton.

ment-Orkos, la couleur reconnaissable des quartes parallèles qu'il hérite des polyphonies primitives du Caucase et de l'Épire. La technique des micro-intervalles recherchent elle aussi une prosodie faisant allusion à l'antiquité.

Ces pages de Serment-Orkos (Ex. 4) et Pour Maurice (Ex. 5) de la même époque, pour baryton et piano, montrent un autre niveau prosodique : le chanteur entonne avec conviction un texte qui n'a aucun sens, puisque constitué de «a» et de «o », sans intertextualité apparente. Betsy Jolas parlait de «musique sous les mots", il faut ici parler de "mots sous la musique», si l'on souhaite comprendre un sens supposé derrière le discours purement sonique de la voix de basse, jamais "lyrique», privée de vibrato ${ }^{14}$, archaïque. Serment rappelle un autre point : Xenakis possède un style vocal assez reconnaissable (comme Maurice Ohana ou Felix Ibarrondo ont le leur), marqué par une certaine rudesse. Xenakis demande des «raclements de gorge rude», des inspirations/ expirations "violentes ne produisant pas de voyelles", des «soupirs très rudes". Même chose dans N'Shima, qui signifie en hébreu «souffle», et utilise des voix "paysannes» de deux mezzo-sopranos, elles aussi sans vibrato, des voix non travaillées au conservatoire : travail du souffle! D’ailleurs, l'instrumental non plus n'a pas le droit de "chanter» : le vibrato est proscrit par exemple pour l'exécution de Thalleïn (1984) pour ensemble. Le vibrato renvoie à une pratique dite évoluée - souvent assimilée au monde de l'opéra - et donc sophistiquée, sentimentale, décadente aux yeux de Xenakis, mais cette hypothèse peut être

et non parallèles. En finale p. 38, la mélodie est réduite à trois sons suivant le même procédé mais simplifié.» (Xenakis, préface à la partition de Procession vers les eaux claires.)

14 Ce n'est pas la seule partition! Dans la musique instrumentale, Jonchaies (1977) ou Thalleïn (1984), «le vibrato est proscrit» (inscription sur la première page). 
mise en question, les voix traditionnelles possèdent elles-mêmes des formes de vibrato! Quoi qu'il en soit, il s'agit d'une quête de vérité première, immédiate et ancestrale, mythique.

Le «retour à l'antique» concerne aussi bien les textes que la mise en musique. Mycènes est l'Atlantide de Xenakis. Heinrich Schliemann a exhumé les neufs couches primitives de Troie; c'est lui également qui révéla ce monde préhellénique mycénien, de l'âge du bronze précédant le monde minoen. Derrière la Grèce d'Eschyle mis en scène dans l'Orestie, se cachent des mythes qui appartiennent à la civilisation mycénienne. Dans le Polytope de Mycènes (1978) déjà évoqué, le spectacle en plein air fait résonner le grec ancien dans le palais royal d'Agamemnon, avec projecteurs anti-aériens sur les collines. Les déclamations en langue mycénienne traduite en grec moderne sont diffusées par des hautparleurs dans toute la vallée.

Xenakis effectue des recherches musicologiques et linguistiques pour Pollo ta dhina (1962, d'après Sophocle, pour chœur d'enfants et orchestre), Medea Senecae [Médée] (1967, d'après Sophocle, chœur d'hommes et quintette), La légende d'Eer (1977, d'après Platon, sons enregistrés), etc., pièces pour lesquelles il utilise les langues d'origine. Les deux œuvres vocales d'après Euripide de 1977 pour chœur : A Hélène et A Colone, suivent «fidèlement» la prosodie de la langue attique du Ve siècle AC (1978/2006a). Même pour l'harmonie, il s'accapare la théorie musicale antique... d'Aritoxène et d'Euclide. Le matériau phonique de Nuits provient en partie du livre Le Déchiffrement des écritures, où se trouve le syllabaire mycénien (1978/2006b, p. 328-333. Doblhofer, 1959, p. 271). «Ce qui m’a passionné dans l'ouvrage de Doblhofer, c'est qu'il montre que tous les alphabets syllabiques étaient basés sur les consonnes et non pas sur les voyelles», explique Xenakis (Julien, 1986, p. 6). D’où les sonorités gutturales et «sauvages» de Nuits entre autres. Au contraire des bruitistes et de l'opinion commune, pour Xenakis la musique émane des consonnes alors que les voyelles constituent des bruits vocaux (ibid.). Ainsi pour N'Shima, «devant chaque voyelle se trouve un son qui vient du fond de la gorge avec des glissandi et des reprises dans le même souffle». Les "parasites» permettent de «salir le son» (ibid.). Les sons $k a, k e, k i$, ko, ku constituent le matériau de Nuits, p. 9, du syllabaire mycénien (Ex. 6) :

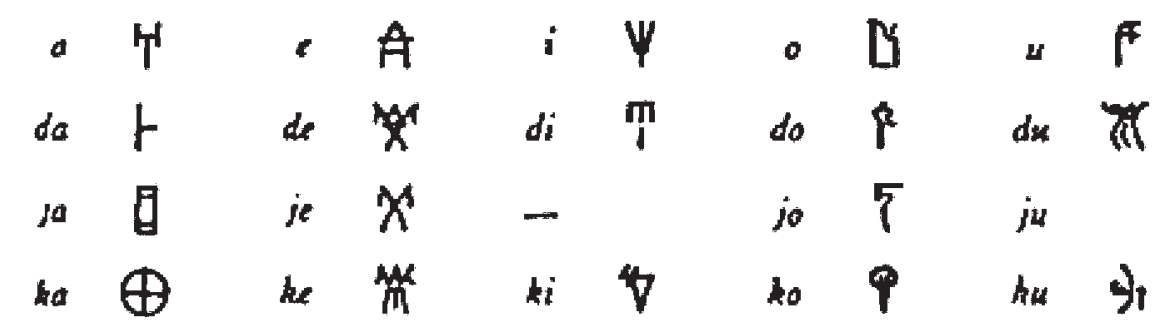

Ex. 6. Syllabaire mycénien d'après John Chadwick, Documents in Mycenaean Greeck (extrait) 
La ritualité et la sacralité antique elle-même « sauvage» (sacrifices humains !) inspirent le compositeur pour la Polytope de Mycènes (1978), qui commence par cette déclamation d'une récitante (Ex. 7):

$$
\begin{aligned}
& \text { W X } \\
& \text { i-je-to-ge di-u-jo do-ra-ge pe-re po-re-na-qe a-ke di-we }
\end{aligned}
$$

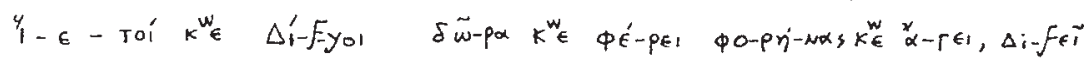

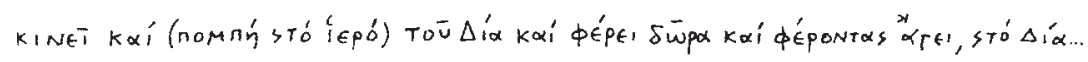

Ex. 7. Ex. 20-Polytope de Mycènes (1978) : début du récit en langue mycénienne

La ligne supérieure est en alphabet mycénien, que Xenakis traduit grâce au syllabaire : «ijetoge diujo donage pere porenage ake diwe». Sa traduction française est la suivante : "Il met en mouvement une procession dans le sanctuaire de Zeus, porte des dons, mène des? [sic], pour Zeus...» (1978/2006b, p. 26).

Or, d'après mes recherches, le fragment de phrase utilisé par Xenakis provient de la tablette mycénienne Pylos PY Tn 316; il s'agit pour être précis de l'avant-dernière phrase (Ex. 8) :

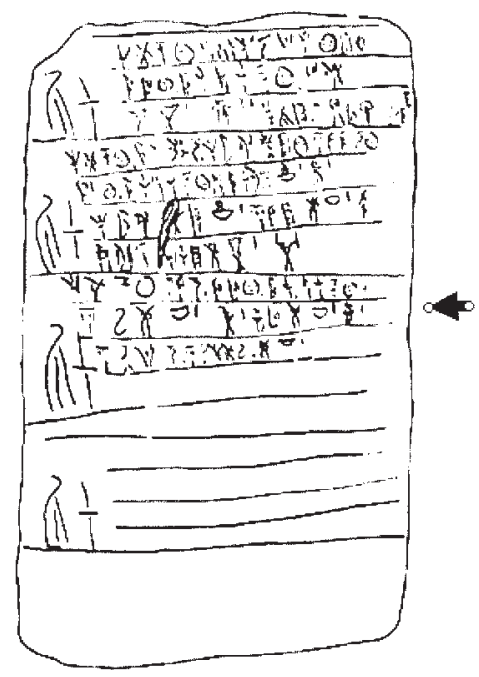

Ex. 8. Tablette mycénienne Pylos PY Tn 316, verso. La flèche indique la phrase utilisée par Xenakis

Bernard Sargent analyse cette tablette mycénienne, qui est pour lui l'une des plus importantes concernant l'héortologie grecque du Ile millénaire (Sergent 1990, p. 175-217). Il donne la traduction de Chadwick (1972, p. 463) : 


$\begin{array}{ll}\begin{array}{l}\text { Puro } \\ \text { ijetoge Diujo donage }\end{array} & \text { [Pylos] } \\ \text { pere porenage } & \text { [sacrifie au sanctuaire de Zeus,] } \\ \text { ake diwe } & \text { [porte des offrandes,] } \\ & \text { [conduit des victimes.] }\end{array}$

Par conséquent, a) il faut ajouter à la traduction de Xenakis ci-dessus : Pylos (ville mycénienne); b) remplacer «met en mouvement une procession» par «sacrifie»; c) enfin, le «? de Xenakis correspond en fait à «victimes». Ne pas garder Pylos peut se comprendre, dans la cadre du Polytope de Mycènes. Pour le reste, voici mon explication : ou bien Xenakis a peiné dans sa traduction, ou bien il a souhaité effacer la référence à la dimension sacrificielle de la cérémonie. La suite de la tablette mycénienne signale que l'offrande est un vase en or et que la victime est une femme.

Bernard Sergent ne définit pas le type de sacrifice, humain ou rite de passage. Mais il faut savoir que les Mycéniens, les Archéens, les Phéniciens, etc. pratiquaient le sacrifice humain. Je ne sais pas de quelle manière Xenakis pouvait l'envisager. La tablette évoque Thésée, Iphigénie, divinités et mythes, comme on le sait, liées à la mer et au sacrifice. Il est probable cette tablette décrive un cycle initiatique féminin, printanier et prénuptial. De jeunes filles sont «amenées dans les sanctuaires, et «données» aux divinités au même titre que des vases en or : c'est-à-dire, sans doute, consacrées pour un service cultuel» (Sergent, op. cit., chap. II). Si l'on replace cette très courte citation dans le contexte du Polytope de Mycènes, nous voyons quelle distance culturelle nous sépare des mycéniens ! La cohérence sociale, artistique, religieuse du rite mycénien est transposée, coupée, insérée dans un «son et lumière » aux thèmes variés, à une époque de grande folklorisation... Xenakis parle quant à lui d'une «réanimation» mêlant l'ancien et le contemporain (1978/2006b). "Je ne veux pas imiter la musique de la Grèce ancienne, mais porter l'esprit de la Grèce ancienne dans la vie» (1991/96, p. 49).

\section{La Crevasse - L'Arbre cosmique}

«L'ordre strict fige et pétrifie : c'est une forme de mort. À la température du zéro absolu, rien ne bouge, rien ne vit. Pour évoluer, il faut créer - mais pour créer, le chaos est nécessaire. La créativité ne peut s'épanouir qu'aux frontières de l'ordre et du chaos. »

(Risset, préface à Darbon, 2006, p. 11)

L'une des traductions du terme Chaos en grec renvoie à l'idée de crevasse (Castoriadis); c'est un gouffre profond, vaste et silencieux (Ovide) ${ }^{15}$. Quand Xenakis s'empare du Dormeur du val d'Arthur Rimbaud, la mise en «abîme» du texte est totale : le titre devient $P u$ Wijnuej we fyp (1992) pour chœur d'enfants, par une application biunivoque de l'alphabet sur lui-même.

15 En grec ancien X $\alpha o \varsigma / K h a o s$, littéralement «Faille, Béance», du verbe $\chi \alpha \imath v \omega / k a i n o ̂$, «béer, être grand ouvert». Dans la philosophie du monde de Castoriadis, l'Être est à la fois Cosmos (ordonné, "déterminé de part en part», et Chaos/Abìme/Sans-Fond, inaccessible à la logique et la métrique. (Castoriadis, 1999. Sorel, 2006). 
La mort est considérée comme une béance noire, avant et après la vie. La religion judéo-chrétienne de la Création et de la Fin diffère de la circularité orientale, l'éternelle renaissance. Le texte sur les supernovae du scientifique Robert P. Kirschner - ces étoiles explosant de façon apocalyptique -, est significatif, parmi ceux choisis pour le Diatope, notamment pour la pensée sur l'infini de Blaise Pascal et les textes énigmatiques de Jean-Paul Richter et d'Hermès Trismégiste, autour de l'errance cosmique et de l'immortalité. ${ }^{16} \mathrm{Le}$ prométhéen Xenakis affronte volontiers la mort; Aïs (1980) pour baryton, percussion et orchestre, est le roi de la mort.

Or, ce terme de faille originelle indique qu'il existait déjà quelque chose de plus ou moins structuré : pas de faille sans structure alentour. Voici apparaître un nouveau mythème, à connotation féminine, maternelle, le vagin de l'enfantement. Et avec lui, non pas une mort-néant, mais une mort grouillant d'une vie potentielle. Quand Xenakis musicalise dans Persephassa (1969), pour six percussions, aux mesures 7-61, les processus entropique/neguentropique (le silence cédant la place à une succession régulière de noires), il traduit cette vision qui parcoure l'humanité d'une évolution du néant vers l'ordre. Comme les grandes pages sur ce thème depuis Hésiode ${ }^{17}$, le chaos initial de la partition - l'introduction est souvent un moment de grande créativité... - finit par s'organiser peu à peu. Le chaos devient rapidement un simple pôle d'une dialectique constructiviste.

C'est alors que l'on ne parle plus de chaos mais de complexié, qui est le rapport dialogique de l'ordre et du désordre (dialogique au sens donné par Edgar Morin). Il est intéressant de noter que parmi les quatre éléments, le Ciel (Ouranos), la Mer (Poséidon) préexistent, forment un chaos d'où surgira la Terre, laquelle offre la stabilité, et, si l'on poursuit, la croissance végétale.

L'œuvre fondatrice du style xenakien, Metastasis (1953-54) pour orchestre, traduit ce mythème génésique ordre/désordre, et en montre les passages. Les carnets de 1951, qui consignent des idées et projets de composition, sont instructifs. Après un "chaos coups répétés dans graves et longs tenus dans aigus», Xenakis prévoit un «inversement ou passage ou autres combinaisons.» (Carnet, 1951,n.n.). Ainsi, «l'ordre doit faire son entrée dans le grand silence subit», quoique dit-il sa «racine» soit «dans le chaos». À la page suivante des Carnets, il esquisse cette structure pour l'œuvre à composer :

«I Toute la richesse cosmique mais chaotiquement

II Naissance de l'ordre qui est très pauvre au début

III La richesse baisse s'apauvrit (sic) mais l'ordre augmente à ses dépens

(...)

IV Complexité de l'ordre en croissance Tout s'ordonne (...)

V Explosion de l'ordre en chaos ordonné»

16 «L'infini» de Blaise Pascal, «Siebenkäs», de Jean-Paul Richter, «Supernovae in other Galaxie» de Robert P. Kirschner. «La composition musicale est à la fois dépendante et indépendante de l'évolution technologique des systèmes analogiques et numériques» (1982/2006, p. 368-369).

17 Ecoutez entre autres Zais (1748) de Jean-Philippe Rameau, Fall And Resurrection (2000) de John Tavener... 


\section{ORDO AD / CUM CHAO ?}

"L'œuvre est faite de formes, le chef-d'œuvre est fontaine informe de formes. L'œuvre est faite de temps, le chef-d'œuvre est source des temps. L'œuvre est un accord sûr, le chef d'œuvre tremble de bruits. »(Serres, 1982, p. 39)

Je terminerai en évoquant un point crucial, celui de l'émergence dont je rappelle le rôle en musique contemporaine dans ma thèse de doctorat [2004]. C'est la notion clef des théories du chaos ou de la complexité (systémique, cognition, etc.). Je l'évoquerai à deux niveaux : dans l'œuvre musicale; dans la vie du compositeur.

La notion est liée à celle d'illusion sonore. Les événements globaux sont faits de milliers de sons isolés; or leur multitude remarque Xenakis peut créer «un événement sonore nouveau sur un plan d'ensemble» (1962/1994, p. 57). Bettina Skrzypczak observe que, dans Pithoprakta (p. 44), l'arrivée «dans le déroulement sonore d'éléments porteurs d'une qualité nouvelle est ressentie comme un tournant critique» (Skrzypczak, 1999, p. 16-21). ${ }^{18}$ Ce peut être une note stationnaire au milieu d'un faisceau de glissandos. "Un autre point critique résulte de l'amoncellement de petites sections de caractère différent, qui survient souvent en même temps que la densité diminue ${ }^{19}$. Il se forme alors des «îles», qui attirent justement l'attention du fait de leur singularité - et du fait de la faible densité. » (ibid.) ${ }^{20}$ C'est ce qu'exprime Georg Lukács : l'informité du Chaos ne peut nous apparaître que par rapport à une forme; la conscience naît de la surprise : "Ce n'est que dans ce qui a reçu une forme que l'on éprouve la métaphysique de l'amorphe : on ressent que le chaos est le principe universel. » (Lukács, 1911, p. 317)

Dans le parcours de Xenakis, il existe un point de bifurcation symbolique et fondamental. Il se réalise pendant la gestation de son cycle vocal des Anastenaria (1953). Cérémonie elle-même incroyable, une mise en transe collective, très ancienne, encore pratiquée au cœur de l'Europe cartésienne. Voici l'«argument» :

Anastenaria est un morceau vivant de civilisations vécues autrefois et arraché à la destruction des millénaires par des paysans grecs de la Thrace. Culte constitué en un ensemble achevé à l'époque de l'Empereur Constantin le Grand, 4e siècle après J.C., et conservé à l'état primitif jusqu'à nos jours. En gros, trois couches rituelles superposées et assimilées lui donnent son caractère extrêmement complexe et extraordinaire, à la manière des cités détruites et rebâties plusieurs fois au cours des temps. La première couche, avec le sacrifice du taureau, la plus primitive, remonte aux temps totémiques et prédéistes. La seconde, avec la danse extatique sur le feu (Ex. 28) se rattache aux cultes agraires et dionysiaques de l'Antiquité grecque ainsi qu'aux cultes asiatiques et thraco-phrygiens d'Héraclès-Sardanapale

\footnotetext{
18 C'est moi qui souligne.

19 Pithoprakta, p. 16 de la partition.

20 C'est moi qui souligne.
} 
et d'Artémis Péracia. La troisième, avec les icônes et l'intervention des prêtres, se rattache au christianisme. Le culte est célébré au mois de mai. ${ }^{21}$

Au moment de cette composition, Xenakis est encore jeune. Il est rempli d'un bouillant mélange de recherches et d'hésitations techniques, esthétiques, une marmite de complexité intérieure. C'est alors que surgit Metastasis, son œuvre phare! J'en ai donné les esquisses tirées des Carnets plus haut. C'est un changement radical, un Big Bang. Inutile de rappeler que la pièce révolutionne l'écriture musicale, passant d'une musique avec des notes à une poétique personnelle, inattendue, holistique, une pensée supramusicale (c'est-à-dire musicale, mais torsadée par les composants mathématiques, visuels, textuels, etc.). Elle va non seulement inaugurer un nouveau monde sonore pour lui, mais marquer son époque. François-Bernard Mâche pense que Metastasis, qui est instrumental, devait constituer le troisième volet d'Anastenaria, un volet vocal. Mais l'œuvre a pris son autonomie, elle s'est séparée du tronc initial; issue de la textualité, elle a pris le visage sublimé de la musique «pure».

Pourquoi cette fulgurance? Le Chaos réhabilite la notion du génie, dont l'analogie avec l'émergence est grande, que ne nie pas Xenakis lui-même, convaincu de l'importance de la créativité (1984/1944,106). Si l'homme est capable de définir des règles, il peut aussi en créer d'autres, non observées, nouvelles. "C’est cela qui constituerait un niveau supérieur de créativité.» (ibid., p. 107) La complexité rend possible l'émergence; l'homme peut créer un «univers différent», qui sera à son tour «une masse infinie de règles dûment enchevêtrées». Car ce que souhaite réaliser le compositeur est «engendrer l'inengendré» (ibid., p. 108).

Pour résumer, Xenakis acte "l'identité interne du monde et du chaos» (Deleuze, 1968/2000). Il est épris comme bien des contemporains du désir de la source originelle. Son territoire, son Tibre, son Euphrate, ce sont la Vénus néolithique et l'Ouranos indo-européen. Le Xaoৎ orageux irradie son visage. Son énigmatique puissance, nous pourrions la comparer aux égyptiens Seth ou Hapy, ces absolus tout à la fois du Déluge et de la Fertilité : tout dépend de notre capacité à en jouer; ils sont l'énergie, la crue, le feu. À l'artiste le pouvoir magique de la féconde catastrophe!

\section{RÉFÉRENCES}

Antunes Jorge. 2001. "Xenakis, Rimbaud und ich Pu Wijnuej we Fyp und Rimbaudiannisia MCMXCV», MusikTexte n 89 : 43-45.

Bachelard, Gaston. 1961. La Poétique de l'espace. Paris : PUF.

Barthel-Calvet, Anne-Sylvie. 2005. «La vitesse, mesure de la continuité sonore chez Xenakis", Definitive Proceedings of the "International Symposium Iannis Xenakis", sous la dir. de Georgaki, Anastasia, Makis Solomos et Giorgos Zervos. Actes du colloque d'Athènes. S.l. s.n.

Castoriadis, Cornelius. 1999. Les carrefours du labyrinthe VI. Paris : Le Seuil. Caullier, Joëlle. 1988. "Pour une interprétation de Nuits», Entretemps n6 : 59-68.

21 Xenakis, préface de la partition Procession aux eaux claires. C'est moi qui souligne. 
Chadwick, John. 1972. Documents in Mycenaean. Londres : Cambridge University Press.

Couroux, Marc. 1994. «Dompter la mer sauvage : réflexions sur Evryali de Iannis Xenakis», Espace Xenakis, sous la dir. de Serge Provost, Circuits, vol. $5, \mathrm{n}^{\circ} 2: 55-67$.

Darbon, Nicolas. 2006. Les musiques du Chaos. Paris : L’Harmattan, coll. «Sémiotique et philosophie de la musique».

—_. 2007a. Musica Multiplex: dialogique du simple et du complexe en musique contemporaine. Paris : L'Harmattan, coll. «Sémiotique et philosophie de la musique».

__ 2007b. «L'Antiorphisme et la technologie musicale (Bruit, chaos, désordre, entropie)», Analyse musicale : 94-103.

—_ 2008. "Pour une anthropologie musicale du contemporain». Dossier d'habilitation à diriger des recherches, Université de Rouen.

___. 2013. "Musicologie, postmodernité, complexité», Musique et Complexité. Autour d'E. Morin et de J-Cl. Risset, sous la dir. de Nicolas Darbon, actes du colloque de décembre 2008. Paris : CDMC, Université de Valencia (Espagne) : Éditions Rivera.

Deleuze, Gilles [1968].200o. Différence et Répétition. Paris : PUF, coll. «Epiméthée».

Doblhofer, Ernest. 1959. Le Déchiffrement des écritures, trad. française de Monique Bittebierre. Paris : Arthaud, coll. «Signes du temps».

Durand, Gilbert. 1984. Les structures anthropologiques de l'imaginaire. Paris : Dunod, Bordas.

Exarchox Dimitris (ed.), Proceedings of the Xenakis International Symposium, Londres, S.l., s.n www.gold.ac.uk/ccmc/xenakis-international-symposium/ programme

Ferapontova Elena V. 2008/11. «Vokalnaya musuka Iannisa Xenakisa kak fenomen ego kompositorskogo tvorchestva» [La musique vocale de Xenakis comme phénomène de sa créativité], thèse de doctorat, Conservatoire d'État Tchaikovsky, publiée sous le titre : Iannis Xenakis. Créativité vocale, [En russe]. Moscou : Éditions Composer.

Flint, Ellen R. 1993. "Metabolae, arborescences and the reconstruction of time in Iannis Xenakis' Psappha». Contemporary Music Review vol. 7, $\mathrm{n}^{\circ} 2: 221-248$.

Frisius, Rudolf. 2003. «Musical et extramusical chez Xenakis. À propos de Kraanerg et de Nekuïa». Iannis Xenakis, Gérard Grisey, la métaphore lumineuse, sous la dir. de Makis Solomos, 193-212.Paris : L'Harmattan.

Georgaki, Anastasia, Makis Solomos et Giorgos Zervos. 2005. Definitive Proceedings of the "International Symposium Iannis Xenakis", actes du colloque d'Athènes. S.l., s.n.

Gerahrds, Hugues (dir.) [1978/1981]. Regards sur Iannis Xenakis. Paris : Stock.

Gill, Dominic. 1978/1981. «Le Polytope de Mycènes». The Financial Times, 14 nov 1978, rééd. Regards sur Iannis Xenakis, 294-298. Paris : Stock

Harley, James. 2004. Xenakis, His life in music. Londres : Routledge. 
_.2011. «Nonlinear Mosaic Form in Kraanerg by Iannis Xenakis». Actes du colloque Xenakis International symposium, sous la dir. de Dimitris Exarchos, http://www.gold.ac.uk/cmru/xenakis-international-symposium/ programme/

Hoffmann Peter. 1994. Amalgam aus Kunst und Wissenschaft. Naturwissenschaftliches Denken im Werk von Iannis Xenakis, 145-152. Francfort : Peter Lang.

2009. Music out of Nothing? Dynamic Stochastic Synthesis: A Rigorous Approach to Algorithmic Composition by Iannis Xenakis. Thèse de doctorat, Université de Berlin, disponible sur : http://opus.kobv.de/tuberlin/volltexte/2009/2410/pdf/hoffmann_peter.pdf. (consulté le 30 avril 2012).

- 2002. "Towards an 'automated Art': Algorithmic Processes in Xenakis' Compositions». Xenakis studies : in memoriam, Contemporary Music Review 21, $\mathrm{n}^{\circ} 2-3$, sous la dir. de John Harley, 121-131. Oxford : Routledge.

Iliescu, Mihu . 2000. "Xenakis et Thom : une morphodynamique sonore». Les Cahiers Arts et Sciences de l'Art, $\mathrm{n}^{\circ} 1: 183-204$.

Julien, Jean-Rémy. 1986. "Nuits d'Iannis Xenakis. Eléments d'une analyse». L'Education musicale $\mathrm{n}^{\circ} 325$.

Le Guay, Patricia. 1992. «Pu wijnuej we fyp, le difficile assemblage du puzzle. Entretien avec Denys Dupays». Mélomane n ${ }^{\circ}$, 5. Paris : Publication de Radio France.

Lukács, Georg [1911]. Die Seele und die Formen. Berlin : Egon Fleischel \& Co

Mâche, François-Bernard. 200o. Un demi-siècle de musique... et toujours contemporaine. Paris : l'Harmattan.

_. 2012. «Discours prononcé par M. François-Bernard Mâche en hommage à Iannis Xenakis lors de la séance publique du mercredi 3 mars 2004", http://www.academie-des-beaux-arts.fr (rubriques : Membres, Composition musicale), reproduit sous le titre «Eloge de Xenakis» sur le site Internet de l'Association des Amis de Xenakis : http://www.iannis-xenakis.org (consulté le 30 avril 2012).

Maffesoli, Michel. 1985. La Connaissance ordinaire. Précis de sociologie compréhensive. Paris : Méridiens.

—.1996/2005. Éloge de la raison sensible. Paris: La Table Ronde.

Matossian, Nouritza. 1981/2005. Iannis Xenakis, rééd. Xenakis. Lefkossia : Moufflon Publications Ltd.

_ 1987]. «Xenakis, master of chaos». Echoes of Le Corbusier, programme de festival, Londres.

Morin, Edgar. 1973. Le paradigme perdu : la nature humaine. Paris: Le Seuil.

Ovide [Publius Ovidius Naso].199oa. Les Fastes, livre I, vol. III, Ier s. AC. Paris : Les Belles Lettres (réed.).

Ovide [Publius Ovidius Naso]. Les Métamorphoses, vers 30-31, env. 8-17 après JC.

Philippot, Michel. 1985. «Xenakis». Encyclopaedia Universalis, tome 18:1131-1135.

Prost, Christine [1981], «Sur l'Orestie ». Regards sur Iannis Xenakis, sous la dir. de Hugues Gerhards, 279-281. Paris : Stock. 
Raanan, Ruth Béatrix. 2001. «Le souffle et le texte : deux approches formelles convergentes». N’Shima de Iannis Xenakis». Présences de Iannis Xenakis, sous la dir. de Makis Solomos, 173-178. Paris : CDMC.

Restagno, Enzo (dir.). 1988. Xenakis. Turin : EDT / MUSICA, coll. «Autori vari».

. 2005. "I Oresteia» / «The Oresteia», Oresteia» Xenakis, akoulouthia Aischylou / Xenakis' Oresteia, Aeschylus Suite, 128-132. Athènes : Ergastirio fonitikis Technis kai erevnas.

Sakkas, Spyros. 2005. "Oresteia», in «Oresteia» Xenakis, akoulouthia Aischylou / Xenakis' Oresteia, Aeschylus Suite, 22-24, 25-27. Athènes : Ergastirio fonitikis Technis kai erevnas,

__ 2010. "Singing... Interpreting Xenakis». Performing Xenakis, 303-334. Hillsdale, New York : Pendragon Press.

Sergent, Bernard. 1990. «Héortologie du mois Plowistos de Pylo», Dialogues d'histoire ancienne, vol. 16, $\mathrm{n}^{\circ} 1: 175-217$.

Serres, Michel. 1982. Genèse. Paris : Grasset.

—. 2011. Musique. Paris : Le Pommier.

Skrzypczak, Bettina. 1999. "De la nécessité d'avoir un système et de ne pas en avoir. La forme en tant que processus chez les premiers Romantiques et chez Iannis Xenakis». Dissonance, Revue Musicale Suisse, n 62 : 16-21.

Solomos, Makis. 1996. Iannis Xenakis. S.l. : P.O. Éditions.

- 2002. «Ta Anastenaria tou Xenaki. Mia paradeigmatiki tomi» («Les Anastenaria de Xenakis. Une rupture paradigmatique»), Mousikos Logos $\mathrm{n}^{\circ}$ 4, rééd. «Les Anastenaria de Xenakis. Continuité et discontinuité historique», disponible sur le site Internet de l'Association des Amis de Xernakis : http://www.iannis-xenakis.org (consulté le 30 avril 2012).

—_. 2003. Iannis Xenakis, Gérard Grisey. La métaphore lumineuse. Paris : L'Harmattan.

.2006. "Cellular automata in Iannis' music. Theory and Pratice», Definitive Proceedings of the "International Symposium Iannis Xenakis", actes du colloque d'Athènes de mai 2005, sous la dir. de Anastasia Geogaki, Makis Solomos et Giorgos Zervos, disponible sur le site Internet de l'Association des Amis de Xenakis : http://www.iannis-xenakis.org (consulté le 30 avril 2012).

Sorel, Reynal. 2006. Chaos et éternité. Mythologie et philosophie grecques de l'Origine. Paris : Les Belles Lettres, collection "Vérité des mythes».

Soulele, Andriana. 2011. "From The Suppliants (Hiketides) to the Oresteia A unique relation between the music of Iannis Xenakis and the staging of Alexis Solomos». Proceedings of the Xenakis International Symposium, sous la dir. de Dimitris Exarchos. Ö. S.l., s.n

Thil, Jean-Marie. 1988. «Iannis Xenakis, Nuits (1967-68)». L'Éducation musicale $\mathrm{n}^{\circ} 352: 41-55$.

Vagopoulou, Evaggelia. 2007. «Cultural Tradition and Contemporary Thought in Iannis Xenakis's Vocal Works». Thèse de doctorat, Université de Bristol.

Varga, Balint Andras. 1996. Conversations with Iannis Xenakis. Londres : Faber and Faber. 
Xenakis, Iannis, Carnets, manuscrits, Bibliothèque nationale de France.

_. 1963. «Musiques Formelles». La revue musicale nº 253 et 254, 30. Paris : Richard Masse.

—.1991/96. «Eschyle, un théâtre total», rééd. [1991].Musique et Originalité, 49-57. Paris : Nouvelles Editions Séguier.

- Kéleütha, Ecrits. Paris : L’Arche : 1956/1994. «Théorie des probabilités et composition musicale», 46-53. 1962/1994. "Eléments sur les procédés probabilistes (stochastiques) de composition musicale», 54-66. 1984/1944. «Musiques et originalité», 106-111.

—. 1994. "Creativity». Perspectives on Musical Aesthetics, 158-164. Londres : W.W. Norton and Co.

1996. «Determinacy and Indeterminacy», Organised Sound 1, $\mathrm{n}^{\circ} 3$ : 143-155.

-Musique de l'architecture, textes réunis par Sharon Kanach. Marseille : Parenthèses : 1978/2006a. "Le Polytope de Mycènes», 328-329. 1978/2006b. «Polytope de Mycènes, programme général», 330-333. 1978/2006c. "Geste de lumière et de son", programme du Diatope de Beaubourg, 353-357. 1980/2006. «Esquisse d'autobiographie», 17-25. 1982/2006. «Diatope : une musique à voir», 358-369.

—. Disque Erato STU70529, Xenakis, Iannis, pochette du disque ERATO STU 70529.

Xenakis, Françoise. 1979. Moi j’aime pas la mer. Paris : Balland.

Xenakis, Mâkhi. 2011. «Un père bouleversant», conférence à Montpellier, Festival de Radio France, disponible sur le site Internet de Mâkhi Xenakis : http://www.makhi-xenakis.com/ (actualités) (consulté le 30 avril 2012).

Zeller, Hans Rudolf. 1987. "Xenakis und die Sprache der Vokalität». MusikKonzepte, $\mathrm{n}^{\circ} 54-55: 3-27$.

\section{ABSTRACT}

The mytheme of Chaos is studied through the vocal works of Xenakis. It is representative of the history, the concerns, and the sensibility of the "contemporary" (of the end of the twentieth century): although inspired by Greek antiquity, the treatment of the text oscillates between the poles of pure abstraction and significant expression. Furious, stormy, the Xenakian Chaos can result from trauma, madness, and war, to indicate the state of what has no shape, the abyss, the deluge, the genesis, the perdition, to be a transition, process, to represent the origin or the end of the world. So the composer wanted "to handle abysses" by the soloist or the choir vocality, to find "all the cosmic wealth, but chaotically." This article opens a diptych; the second section is entitled "The Big Neolithic Mother in Serment-Orkos" (published by the Iannis Xenakis Center, University of Rouen). The postulate is that chaos theory and complexity theory belong themselves to an anthropology of the imagination. This article joins research on the transdiction (notion explained on the site of the CEREdI, University of Rouen): sound and body in the musico-literary transfers. 


\section{RÉSUMÉ}

Le mythème du Chaos est étudié à travers les œuvres vocales de Xenakis. Il est représentatif de l'histoire, des préoccupations et de la sensibilité "contemporaines" (de la fin du $\mathrm{XX}^{\mathrm{e}}$ siècle) : bien que s'inspirant de l'Antiquité grecque, le traitement du texte oscille entre les pôles de l'abstraction pure et de l'expression signifiante. Furieux, tempétueux, le chaos xenakien peut résulter du traumatisme, de la folie, de la guerre, désigner l'état de ce qui n'a pas de forme, la béance, le déluge, la genèse, la perdition, être transition, processus, représenter l'Origine ou la Fin du monde. Ainsi le compositeur a-t-il voulu "traiter des abîmes" par la vocalité soliste ou chorale, remonter à "toute la richesse cosmique, mais chaotiquement». Cet article ouvre un diptyque dont le second volet s'intitule "La Grande Mère néolithique dans Serment-Orkos» (publié par le Centre Iannis Xenakis, Université de Rouen). Le postulat est le suivant : les sciences dites du «chaos» et de la «complexité» relèvent elles-mêmes d'une anthropologie de l'imaginaire. Par ailleurs, il s'inscrit dans un champ de recherche sur la transdiction (notion explicitée sur le site du CEREdI, Université de Rouen), c'est-à-dire sur le son et la corporéité des transferts musico-littéraires. 